\title{
Antidiabetic and Hypolipidemic Effects of Different Fractions of Catharanthus Roseus (Linn.) on Normal and Streptozotocin-induced Diabetic Rats
}

\author{
M. A. Islam, M. Afia Akhtar, M. R. I. Khan, M. S. Hossain, M. K. Alam, M. I. I. Wahed, \\ B. M. Rahman, A. S. M. Anisuzzaman, S. M. Shaheen, and Maruf Ahmed ${ }^{1}$ \\ Department of Pharmacy, Rajshahi University, Rajshahi-6205, Bangladesh
}

Received 29 August 2008, accepted in final revised form 11 February 2009

\begin{abstract}
The antidiabetic and hypolipidemic effects of petroleum-ether, ethyl acetate and chloroform fractions from ethanolic extract of the leaves of Catharanthus roseus ( $C$. roseus) were investigated in normal and streptozotocin-induced diabetic rats (SIDRs). Single doses $(150 \mathrm{mg} / \mathrm{kg}$, i.p.) of $C$. roseus extracts in the fasting blood glucose (FBG) levels were determined in normal and SIDRs on $0,1,2,3,6,10,16$, and $24^{\text {th }}$ hours and serum triglyceride (TG) and serum total cholesterol (TC) levels were determined after $24^{\text {th }}$ hour. In normoglycemic rats and in SIDRs, petroleum-ether and ethyl acetate fraction of C. roseus reduced blood glucose level significantly. In case of hypolipidemic effects, all fractions reduced serum total cholesterol but the ethyl acetate fraction of C. roseus was the most effective. All fractions of $C$. roseus reduced serum triglyceride level but the ethyl acetate fraction reduced triglyceride level at the highest. The antidiabetic and hypolipidemic activities were compared to metformin $\mathrm{HCl}(150 \mathrm{mg} / \mathrm{kg})$. Of all the three fractions, ethyl acetate fractions were the best in activity. Ethyl acetate fraction of $C$. roseus was found to contain flavonoids and alkaloids. The mechanism underlying the antidiabetic activity is probably increased glycogenesis, decreased gluconeogenesis or decreased absorption of glucose from intestine.
\end{abstract}

Keywords: Catharanthus roseus; Antidiabetic; Hypolipidemic; Streptozotocin-induced diabetic rat.

C 2009 JSR Publications. ISSN: 2070-0237 (Print); 2070-0245 (Online). All rights reserved. DOI: $10.3329 /$ jsr.v1i2.1075

\section{Introduction}

Diabetes mellitus is a metabolic disorder characterized by hyperglycemia, abnormal lipid and protein metabolism along with specific long-term complications affecting the retina, kidney and nervous system [1]. Hyperglycemia is an important factor in the development and progression of the complications of diabetes mellitus [2]. The pathogenesis of diabetes mellitus is managed by insulin and oral administration of hypoglycemic drugs such as sulfonylureas and biguanides [3]. Unfortunately, apart from having a number of side effects, none of the oral synthetic hypoglycemic agents has been successful in

${ }^{1}$ Corresponding author: amaruf2000@yahoo.com 
maintaining euglycaemia and controlling long-term microvascular and macrovascular complications [3-5]. The toxicity of oral antidiabetic agents differs widely in clinical manifestations, severity, and treatment [6]. The use of herbal medicines for the treatment of diabetes mellitus has gained importance throughout the world. The World Health Organization also recommended and encouraged this practice especially in countries where access to the conventional treatment of diabetes is not adequate [7]. There is an increased demand to use natural products with antidiabetic activity due to the side effects associated with the use of insulin and oral hypoglycemic agents [8, 9]. The available literature showed that there are more than 400 plant species having hypoglycemic activity [10-12]. Though some of these plants have great reputation in the indigenous system of medicine for their antidiabetic activities, many remain to be scientifically established. Hypercholesterolemia and hypertriglyceridemia are common complications of diabetes mellitus in addition to hyperglycemia [13-15]. The frequency of hyperlipidemia in diabetes is indeed very high, depending on the type of diabetes and its degree of control [16].

Periwinkle Catharanthus roseus Linn. (Nayantara) is an herbaceous ever-blossoming sub-shrub, grown as an ornamental plant in gardens all over Bangladesh. It has been reported that the juice of fresh leaves of Catharanthus reduces blood glucose in normal and alloxan-induced diabetic rabbits [17]. B. Antia and J. Okonon have demonstrated significant reduction in the levels of total cholesterol, triglycerides, LDL (low-density lipoprotein) and VLDL (very low-density lipoprotein) cholesterol using the fresh leaf juice of catharanthus in normal rats [18]. Leaf extract of Catharanthus is also shown to have blood glucose decreasing property [19]. S. Satyanrayana and colleagues have shown the leaf juice of Catharanthus and the seed powder of fenugreek have their hypoglycemic action individually and in combination on rabbits [20].

Some research have been performed on ethanolic crude extract of the plant yet the glucose lowering effects of petroleum ether, chloroform and ethyl acetate fractions isolated from the ethanolic extract have not been done. This fractionation effects will help us to determine which fraction is more potent. This finding will indicate which compounds are actually responsible for antidiabetic properties. In this study the effects of petroleum ether, ethyl acetate and chloroform extracts isolated from ethanolic extracts of C. roseus on fasting blood glucose (FBG) and lipid biochemical parameters such as serum total cholesterol (TC) and serum triglyceride (TG) were investigated on normal and SIDRs where metformin-HCl is used as standard drug. Thus the hypoglycemic, antihyperglycemic and hypolipidemic effects of the plant has been investigated.

\section{Materials and Methods}

\subsection{Plant material}

Fresh leaves of $C$. roseus were collected from the medicinal plant garden of our University and were dried under shadow for several days. The dried leaves were then ground to a coarse powder. The authenticity of the C. roseus was identified by Mr. A.H.M. Mahbubur Rahman, Department of Botany, Rajshahi University. Voucher 
specimens, collection \# 37, dated 10/30/2000 for C. roseus, kept in the Department of Botany, Rajshahi University, Rajshahi-6205, Bangladesh.

\subsection{Reagents}

Metformin $\mathrm{HCl}$ was the generous gift sample from Square Pharmaceuticals Ltd., Pabna, Bangladesh. Both the streptozotocin- $\mathrm{HCl}$ and DMSO were purchased from Loba Chemie, Bombay, India. DMSO (dimethyl sulfoxide) was used to dissolve metformin and the extracts of $C$. roseus, since these substances are insoluble in water and other available inert solvents [21].

\subsection{Preparation of ethanol extracts}

Dried leaves of C. roseus were soaked for 5-7 days in 2 liter of 95\% ethanol with occasional shaking and stirring. Then, they were passed through cotton and then filtered through filter paper. The remaining parts were filtered again under the same procedure. Then the solvent i.e., ethanol was allowed to evaporate using rotary evaporator at temperature $40-45^{\circ} \mathrm{C}$. Thus the highly concentrated ethanolic extract was obtained.

\subsection{Fractionation of ethanol extract}

Crude rectified spirit extract was diluted by addition of $150 \mathrm{ml}$ distilled water to obtain aqueous solution. The aqueous solution is then treated with $50 \mathrm{ml}$ petroleum ether for three times. The upper fraction was collected in each time of fractionation by using separating funnel. The aqueous fraction is then treated with chloroform $50 \mathrm{ml}$ for three times. The lower fraction was collected for getting chloroform extract. The remaining aqueous fraction was again treated with $50 \mathrm{ml}$ ethyl acetate for three times. The upper fraction was collected for getting ethyl acetate fraction.

The fractions of the different solvents were then evaporated by rotary evaporator. The remaining portions of the different fractions were then dried by using mild sunlight. The dried extracts were then preserved in the freeze for the experimental use [22].

\subsection{Phytochemical screening tests}

The following phytochemical screening methods [23] were used for tests:

Test for saponins: Boiled $300 \mathrm{mg}$ of extract with $5 \mathrm{ml}$ water for two minutes. Mixtures was cooled and mixed vigorously and left it for three minutes. The formation of frothing indicates the presence of saponins.

Test for tannins: To an aliquot of the extract added sodium chloride to make to $2 \%$ strength. Filtered and mixed with $1 \%$ gelatin solution. Precipitation indicates the presence of tannins. 
Test for triterpenes: $300 \mathrm{mg}$ of extract mixed with $5 \mathrm{ml}$ chloroform and warmed for 30 minutes. The chloroform solution is then treated with a small volume of concentrated sulfuric acid and mixed properly. The appearance of red color indicates the presence of triterpenes.

Test for alkaloids: $300 \mathrm{mg}$ of extract was digested with $2 \mathrm{M} \mathrm{HCl}$. Acidic filtrate was mixed with amyl alcohol at room temperature, and examined the alcoholic layer for the pink colour, which indicates the presence of alkaloids.

Test for flavonoids: The presence of flavonoids was determined using $1 \%$ aluminium chloride solution in methanol, concentrated $\mathrm{HCl}$, magnesium turnins and potassium hydroxide solution.

\subsection{Animal experiments}

A total number of 50 long-Evans female rats weighing about $150-180 \mathrm{mg}$ age 2 months were purchased from animal house of International Centre for Diarrhoeal Disease Research, Bangladesh (ICDDR, B). Prior to the commencement of the experiment, all the rats were acclimatized to the new environmental condition for a period of one week. During the experimental period, the rats were kept in a well-ventilated animal house at room temperature of $25^{\circ} \mathrm{C}$ and were supplied with standard pellets supplied from ICDDR, $\mathrm{B}$ and fresh drinking water ad libitum. They were kept in cages and maintained in wellventilated room under conditions of natural light and dark cycle. Animals were fasted for $16 \mathrm{~h}$ prior to drug administration allowing access only to water and were deprived of both food and water during the experiment.

\subsection{Induction of diabetes}

The rats were randomly divided into 10 groups, each containing 5 rats. After fasting $16 \mathrm{~h}$, rats of group (VI-X) were rendered diabetic by injecting intraperitoneally a freshly prepared solution of streptozotocin $(45 \mathrm{mg} / \mathrm{kg})$ in $0.1 \mathrm{~mol} / \mathrm{L}$ citrate buffer, $\mathrm{pH} 4.5$, in volume $1 \mathrm{ml} / \mathrm{kg}$ [24], after a base line glucose estimation was done. After 48 hours blood glucose content was measured by using BioLand G-423 Glucose Test Meter (BioLand, Germany) using Blood sample from the tail vein of the rats. When the condition of diabetes was established animals with blood glucose levels above $11.1 \mathrm{mmol} / \mathrm{L}$ were selected for the study [25].

\subsection{Effect on diabetic rats}

Group I served as a nondiabetic control while group VI for diabetic control group. Group II served as nondiabetic metformin control while group VII served as diabetic metformin controlled group. Group II and VII were treated with metformin $\mathrm{HCl}(150 \mathrm{mg} / \mathrm{kg}$, i.p.) for 24 hours experiment. Group III, IV, V and group VIII, IX and X were treated with the petether, ethyl acetate and chloroform fractions of ethanolic extract of C. roseus at 150 
$\mathrm{mg} / \mathrm{kg}$ for 24 hours experiment. The reference drug and the extracts were administered intraperitonialy to the rats.

\subsection{Collection of blood and serum and determination of blood glucose, serum total cholesterol and serum triglycerides}

Blood samples were collected from tail vein of each rat of a group before and also at 0,1 , $2,3,6,10,16$, and $24^{\text {th }}$ hours of one day experiment. The samples were analyzed for blood glucose content by using BioLand G-423 glucose test meter (BioLand Germany). Then the rats were sacrificed and about 1-2 $\mathrm{ml}$ of blood was collected directly from the heart by syringes, centrifuged at $4000 \mathrm{rpm}$ for 10 minutes and the serum was obtained for the determination of TC and TG. Serum TC and TG concentrations were analyzed by measuring absorbance by UV spectrophotometer (Shimidzu UV-1200, Tokyo, Japan), using wet reagent diagnostic kits (Boehringer Mannheim, $\mathrm{GmbH}$ ) according to manufacturer's protocol.

\subsection{Statistical analysis}

Data were expressed as mean \pm standard error of mean (SEM). Statistical comparisons were performed by one-way ANOVA followed by Dunnett's Multiple Comparison Test (DMCT) and the values were considered statistically significant when $p<0.01$. Statistical calculations and the graphs were prepared using GraphPad Prism version 4.00 for Windows (GraphPad Software, San Diego, CA, USA, www.graphpad.com).

\section{Results and Discussions}

The effects of different fractions of the ethanolic extracts of $C$. roseus on the FBG, serum TC and serum TG levels were investigated in the control and streptozotocin-induced diabetic rats using metformin $\mathrm{HCl}$ as standard antidiabetic agent.

\subsection{Effect of different fractions of $C$. roseus on fasting blood glucose level in normoglycemic rats}

The mean blood glucose concentration of controlled and fractions of C. roseus-treated animals (after intraperitoneal administration of a single dose) on 0, 1, 2, 3, 6, 10, 16, and $24^{\text {th }}$ hours are shown in Fig. 1. Hypoglycemia was observed in animals treated with $C$. roseus extracts. The significant reduction $(p<0.01)$ of $48.34 \%$ for petroleum ether-CR was observed at $10^{\text {th }}$ hour of the experiment. The chloroform extract of $C$. roseus has no significant effect on the blood glucose level. Chloroform-CR fraction showed some toxic effect because some rats were died. This may be due to the presence of some alkaloids with anticancer properties of the plant. The ethyl acetate fraction of $C$. roseus reduced FBG by $40.68 \%$ was observed at $24^{\text {th }}$ hour for ethyl acetate. This may be due to the 
presence of hypoglycemic alkaloids (catharanthin, leurosine, lochnerine, tetrahydroalstonin, vindoline and vindolinine) $(p<0.01)$ [26]. A significant reduction $(p<0.01)$ in blood glucose of $52.55 \%$ was observed for metformin- $\mathrm{HCl}$ at $6^{\text {th }}$ hour after treatment in comparison to control. All treatment is done with a single dose of $(150 \mathrm{mg} / \mathrm{kg}$ body weight).

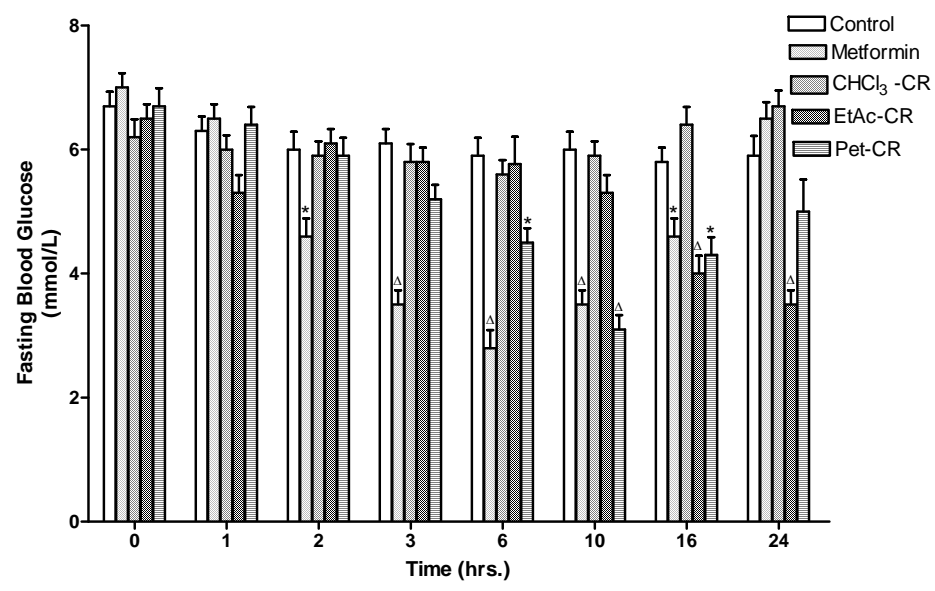

Fig. 1. Effect of different fractions of C. roseus $(150 \mathrm{mg} / \mathrm{kg})$ on Fasting Blood Glucose level (mmol/L) in normal rats. $*$ and $\Delta(* *)$ indicate significant changes in FBG level compared to normal rats after treatment $(p<0.05$, $p<0.01$ respectively). The result is expressed as mean $\pm \mathrm{SEM}$.

\subsection{Effect of different fractions of $C$. roseus on fasting blood glucose level in streptozotocin-induced diabetic rats}

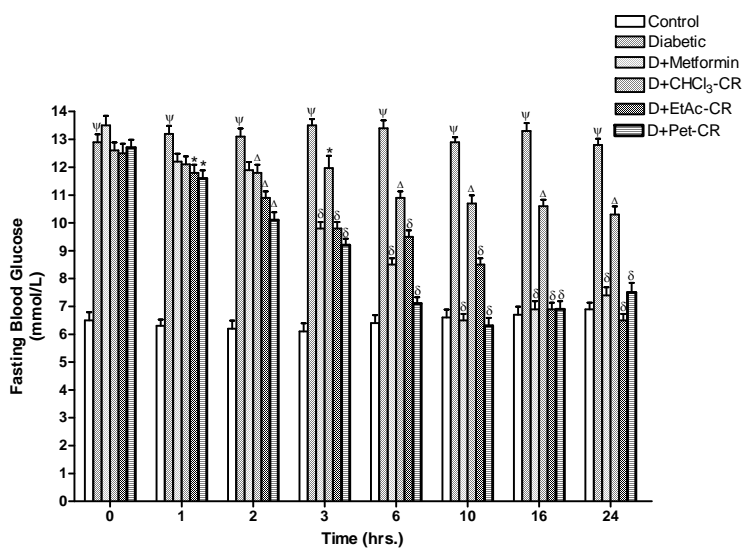

Fig. 2. Effect of different fractions of $C$. roseus on the FBG level on diabetic rats compared to normal rats. $\Psi(* * *)$ indicates significant change in blood glucose level compared with normal control group $(p<0.001) . *$, $\Delta(* *)$ and $\delta(* * *)$ indicate significant changes in FBG level in SIDRs after treatment $(p<0.05, p<0.01$ and $p<$ 0.001 , respectively). The result is expressed as mean $\pm \mathrm{SEM}$. 
The mean blood glucose concentration of controlled and fractions of $C$. roseus-treated animals (after intraperitoneal administration of a single dose) on $0,1,2,3,6,10,16$, and $24^{\text {th }}$ hours are shown in Fig. 2. Hypoglycemia was observed in animals treated with $C$. roseus extracts. The significant reduction $(p<0.001)$ of $51.67 \%$ for petroleum ether-CR occurs at $10^{\text {th }}$ hour of the experiment. Ethyl acetate-CR fraction showed maximum reduction $(p<0.001)$ of $49.22 \%$, observed at $24^{\text {th }}$ hour. Metformin caused maximum reduction $(p<0.001)$ of blood glucose level of $49.62 \%$ on $10^{\text {th }}$ hour of experiment after treatment in comparison to controlled diabetic rats. All treatment is done with a single dose of (150 mg/kg body weight).

\subsection{Effects of different fractions of Catharanthus roseus on total cholesterol and triglyceride levels in normal rats}

The mean serum total cholesterol and triglyceride levels of control and extracts of $C$. roseus -treated animals (after intraperitoneal administration of a single dose) on the $24^{\text {th }}$ hour are shown in Fig. 3 and Fig. 4. Hypolipidemia was observed in animals treated with C. roseus extracts.

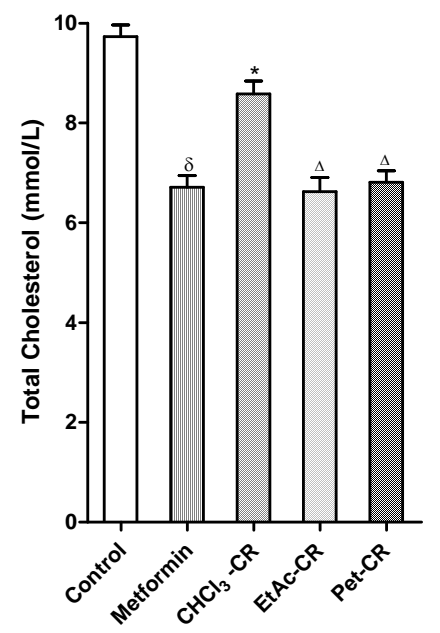

Fig. 3. Effect of different fractions of $C$. roseus on the total cholesterol $(\mathrm{mmol} / \mathrm{L})$ in normal rats. $*, \Delta(* *)$ and $\delta(* * *)$ indicate significant change in serum total cholesterol compared with normal control group $(p<0.05)$. The result is expressed as mean \pm SEM.

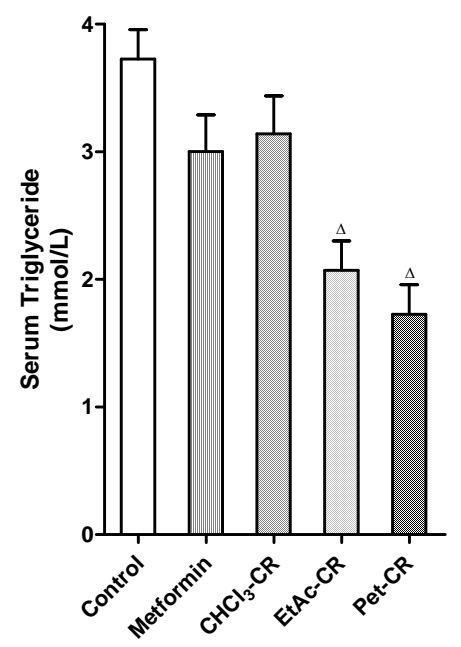

Fig. 4. Effect of different fractions of $C$. roseus on the triglyceride $(\mathrm{mmol} / \mathrm{L})$ in normal rats. $\Delta(* *)$ indicates significant change in serum triglyceride compared with normal control group $(p<0.01)$. The result is expressed as mean $\pm \mathrm{SEM}$. 
During the effect of different fractions of C. roseus (Fig. 3) on the cholesterol level of normal rats $\mathrm{CHCl}_{3}-\mathrm{CR}$, ethyl acetate-CR and pet ether-CR fractions decreased cholesterol level to $87.46 \%$ (not significant), $68.03 \%$ and $69.99 \%$ respectively. So the ethyl acetateCR fraction showed maximum reduction of $31.97 \%$, which is similar to metformin (31.01\%) also.

During the effects of different fractions of C. roseus (Fig. 4) on serum triglyceride level of normal rats the chloroform-CR, ethyl acetate-CR and petroleum ether-CR showed serum triglyceride level of $73.11 \%, 55.64 \%$ and $60.48 \%$ respectively. Maximum reduction of serum triglyceride level of $44.36 \%$ was observed for ethyl acetate-CR.

\subsection{Effects of C. roseus on total cholesterol and triglyceride levels in streptozotocin- induced diabetic rats}

During the effects of different fractions of C. roseus (Fig. 5) on SIDRs $\mathrm{CHCl}_{3}-\mathrm{CR}$, ethyl acetate-CR and pet ether-CR showed total cholesterol level of $68.81 \%, 64.33 \%$ and $68.28 \%$ respectively where metformin reduces $64.61 \%$. Maximum reduction of $35.67 \%$ was observed for ethyl acetate-CR., which is also similar to metformin action.

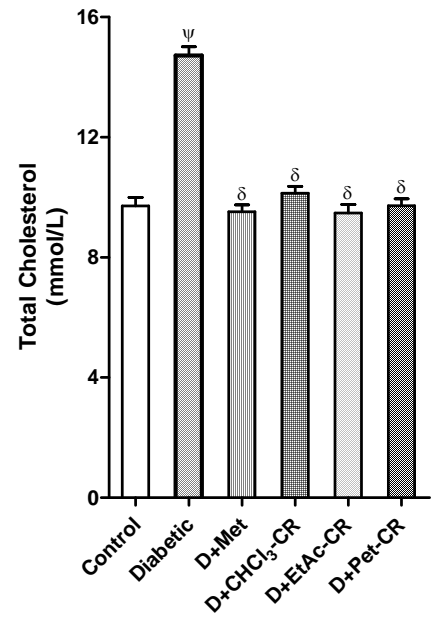

Fig. 5. Effect of different fractions of $C$. roseus on the serum total cholesterol $(\mathrm{mmol} / \mathrm{L})$ on diabetic rats compared to normal rats. $\psi(* * *)$ indicates significant change in total cholesterol of diabetic group compared with normal control group ( $p<$ $0.001) . *, \Delta(* *)$ and $\delta(* * *)$ indicate significant changes in total cholesterol compared with diabetic control group $(\mathrm{p}<$ $0.05, p<0.01$ and $p<0.001$ respectively). The result is expressed as mean \pm SEM.

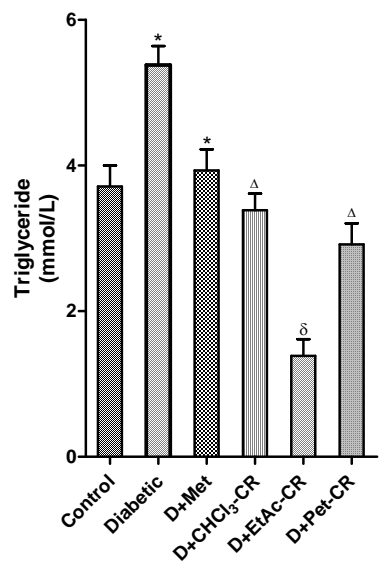

Fig. 6. Effect of different fractions of $C$. roseus on the serum triglyceride $(\mathrm{mmol} / \mathrm{L})$ on diabetic rats compared to normal rats. $\psi(*)$ indicates significant change in serum triglyceride compared with normal control group $(p<0.05)$. * $\Delta(* *)$ and $\delta(* * *)$ indicate significant change in serum triglyceride compared with diabetic control group ( $p<0.05, p<$ 0.05 and $p<0.05$ respectively). The result is expressed as mean \pm SEM. 
During the effects of different fractions of C. roseus (Fig. 6) on serum triglyceride level of normal rats the $\mathrm{CHCl}_{3}-\mathrm{CR}$, ethyl acetate-CR and petroleum ether-CR showed serum triglyceride level of $73.11 \%, 55.64 \%$ and $60.48 \%$, respectively where metformin reduces to $80.64 \%$. Maximum reduction of serum triglyceride level of $44.36 \%$ was observed for ethyl acetate-CR.

However, the serum total cholesterol and triglyceride lowering efficiency of ethyl acetate fraction of $C$. roseus was found higher than other fractions. All the plant extracts were found to have the antihyperlipidemic activity in SIDRs.

\subsection{Phytochemical screening test result}

The phytochemical screening test result is shown in the Table 1.

Table 1. Chemical constituents of different fractions of C. roseus

\begin{tabular}{lccccc}
\hline & Saponin & Tanins & Triterpines & Alkaloids & Flavonoids \\
\cline { 2 - 6 } Petroleum ether- & - & + & - & + & + \\
CR & & & & & + \\
Chloroform-CR & - & - & - & + & + \\
Ethyl acetate-CR & - & - & - & + \\
\hline \multicolumn{2}{c}{$(-)-$ Not detected; $(+)$ - Detected }
\end{tabular}

In the mid -1960s streptozotocin was found to be selectively toxic to the $\beta$-cells of the pancreatic islets, the cells that normally regulate blood glucose levels by producing the hormone insulin. This suggested the drug's use as an animal model of type I diabetes [27]. It is well established that biguanides like metformin produce hypoglycemia by increasing the secretion of insulin from the pancreas [28] and these compounds are active in mild Streptozotocin-induced diabetes whereas they are inactive in intense streptozotocin diabetes (nearly all $\beta$-cells have been destroyed). However, since our results showed that metformin reduced the blood glucose levels in hyperglycemic rats, the state of diabetes is not severe. Streptozotocin-treated animals receiving the extracts of $C$. roseus showed rapid normalization of blood glucose levels in comparison to the control and this could be due to the possibility that some $\beta$-cells are still surviving to exert their insulin releasing effect by different fractions of C. roseus. No histological studies were carried out to prove this and it is not possible to explain the detailed mechanism of antidiabetic action of the fractions of $C$. roseus. The active principles in the $C$. roseus, which may be responsible for the hypoglycemic and hypolipidemic actions, are unknown. This may be due to the presence of hypoglycemic alkaloids (catharanthin, leurosine, lochnerine, tetrahydroalstonin, vindoline and vindolinine) [26]. This suggests that the the plant extracts studied in this protocol might have bound to insulin receptors to act as insulin secretagogue, like biguanides. Other probable mechanisms by which the extracts of $C$. roseus lowered blood glucose levels in diabetic rats might be by increasing glycogenesis, inhibiting gluconeogenesis in the liver, or inhibiting the absorption of glucose from the intestine or these might have improved insulin resistance. Further experiments are needed 
to determine the actual mechanism of action of the active constituents of the relative plant fractions. In the present study, there was a significant reduction in the levels of total cholesterol and triglycerides. This reduction could have resulted from the antioxidant effect of the different fractions of ethanol extract of C. roseus, whose phytochemical components include flavonoid, which is known for antioxidant effect. Further investigations are warranted to identify the hypolipidemic mechanism of the active principles in C. roseus.

\section{Conclusion}

Plant medicines (phytotherapies) have a long history as treatment for diabetes. With a disturbing rise in the prevalence of this metabolic disease and associated healthcare costs, interest in alternative or complementary therapies has grown. Our study have shown that the ethyl acetate fraction of ethanolic extract of $C$. roseus is most effective in glucose lowering effect in normal and streptozotocin-induced hyperglycemic rats. In the present study, there was a significant reduction in the levels of total cholesterol and triglycerides. This reduction could have resulted from the antioxidant effect of the extracts of C. roseus, whose phytochemical components include flavonoid, which is known for antioxidant effect. This also may be due to the presence of hypoglycemic alkaloids (catharanthin, leurosine, lochnerine, tetrahydroalstonin, vindoline and vindolinine) [26]. Our preliminary phytochemical analysis has indicated that flavonoids and alkaloids have been reported to exert potent hypoglycemic and hypolipidemic effects. It needs to be determined as to which components of $C$. roseus are responsible for the hypoglycemic and hypolipidemic activity exhibited by the ethyl acetate fraction. However this study will pave the way for plant based specific treatment of diabetes avoiding the complications of artificial drug substances.

\section{References}

1. M. N. David, M. James, and E. S. Daniel, Lancet 350 (Suppl. 1) S14 (1997). doi:10.1016/S0140-6736(05)64186-4

2. L. Luzi, New Eng. J. Med. 339, 115 (1998). doi:10.1056/NEJM199807093390210

3. A. G. Gilman and L. S. Goodman, The Pharmacological Basis of Therapeutics, 5th Edition (Macmillan, New York, 1985).

4. A. Momin, in Role of indigenous medicine in primary health care - Proc. of First Int. Seminar on Unani Medicine (New Delhi, India, 1987) p. 54.

5. P. H. S. Stenman, K. Groop, E. Laakkonen, E. Wahlin-Boll, and A. Melander, Diabet. 39, 108A (1990).

6. H. A. Spiller and T. S. Sawyer, American J. of Health-System Pharm. 63, 929 (2006). doi:10.2146/ajhp050500

7. WHO Expert Committee on Diabetes mellitus - Technical report series 646. 2nd report, (World Health Organization, Geneva, 1980) pp.1-80.

8. R. R. Holman and R. C. Turner, Textbook of Diabetes (Blackwell, Oxford, 1991).

9. B. Rao. Kameswara, R. Giri and M. M. Kesavalu, Manphar Vaidhya Patrika. 1, 33 (1997).

10. S. K. Mukherjee, J. of the Diabet. Assoc. Ind. 21 (1), 97 (1981).

11. B. Oliver-Bever, Oral hypoglycemic action of medicinal plants in tropical West Africa (Cambridge University Press, 1986). 


\section{Antidiabetic and Hypolipidemic Effects}

12. K. Rai, Ancient Science of Life 14, 42 (1995).

13. S. A. Riyad, A. Ghani, A. Salam, and S. M. Suleiman, Planta Medica 54, 286 (1988). doi:10.1055/s-2006-962434

14. S. P. Tarfa, P. K. Joseph, and K. T. Augusti, Current Science 57, 31 (1988).

15. S. R. Sharma, S. K. Dwivewdi, and D. Swarup, Ind. J. of Exp. Biology 34, 372 (1996).

16. E. O. Balassa, Medicographia 7, 11 (1985).

17. S. Nammi, M. K. Boini, S. Lodagala, and R. B. S. Behara, BMC Complement Altern. Med. 2, 3 (2003).

18. B. S. Antia and J. E. Okonon, Int. J. Pharmacol. 37, 401 (2005).

19. M. Y. Habib, M. S. Islam, and M. A. Khan, Pak. J. of Nutrition 4 (1), 17 (2005). doi:10.1186/1475-2891-4-17

20. S. Satayanarayana, G. S. Sarma, A. Ramesh, K. Sushruta, and N. Srinival, Pharm. Biol. 41 (6), 466 (2003). doi:10.1076/phbi.41.6.466.17830

21. M. A. Akhtar, M. Rashid, M. I. Ibne Wahed, M. R. Islam, S. M. Shaheen, M.A. Islam, M. S. Amran, and M. Ahmed, Res. J. of Med. and Medical Sciences 2 (1), 29 (2007).

22. A. H. M. Khurshid Alam, M. Pharm. Thesis, Rajshahi University (1999).

23. B. S. Nayak, and L. M. Pinto Pereira, BMC Complement Altern. Med. 6, 41(2006). doi:10.1186/1472-6882-6-41

24. O. Siddique, Y. Sun, J. C. Lin, and Y. W. Chein, J. Pharm Sci. 76, 341 (1987). doi:10.1002/jps.2600760416

25. X. C. Liang, M. Z. Xie, and S. S. Guo, Chinese J. Integ. Trad. West med. 13 (10), 587 (1993).

26. G. H. Svoboda, M. Gorman, and M.A Root, Ltoydia. 27, 361 (1964).

27. K. R. Mansford, L. Opie, Lancet 1 (7544), 670 .PMID 4170654 (1968).

28. R. S. Yallow, H. Black, M. Villazan, S.A. Berson, Diabetes 9, 356 (1960). 